\title{
Preliminary Study on Quantitative Sleep EEG Characteristics in Patients with Schizophrenia
}

\author{
Seong Min Oh${ }^{1}$, Yu Jin Lee ${ }^{1}$, Jong Won Kim²,3,4, Jae Won Choi', and Do-Un Jeong ${ }^{1}$ \\ ${ }^{1}$ Department of Psychiatry and Center for Sleep and Chronobiology, Seoul National University Hospital, Seoul, Republic of Korea \\ ${ }^{2}$ Cooperative Research Centre for Alertness, Safety and Productivity, The University of Sydney, Sydney, Australia \\ ${ }^{3}$ Sleep and Circadian Group, Woolcock Institute of Medical Research, Glebe, Australia \\ ${ }^{4}$ School of Physics, The University of Sydney, Sydney, Australia
}

We used quantitative electroencephalography (EEG) spectral analysis to compare activity in the bilateral frontal, central, and occipital areas in nine patients with schizophrenia and ten healthy control subjects during standard nocturnal polysomnography. Patients with schizophrenia had longer sleep latency than controls. In N2 sleep, the patients had significantly lower $0.5-1 \mathrm{~Hz}$ power and higher theta power in the left frontal region, and higher beta power in the left occipital region than did control subjects. In N3 sleep, the patients with schizophrenia had significantly higher alpha power in the left occipital region than did controls. These findings show distinctive EEG sleep patterns in patients with schizophrenia, which may reflect brain dysfunction or medication effects.

Psychiatry Investig 2017;14(2):219-225

Key Words Schizophrenia, Sleep, Electroencephalography.

\section{INTRODUCTION}

Schizophrenia is characterized by marked sleepdisturbances, ${ }^{1}$ such as difficulty in initiating and maintaining sleep, ${ }^{2-5}$ decreased total sleep time, ${ }^{2,5}$ poor sleep efficiency, ${ }^{2,45}$ decreased slow-wave sleep (SWS), ${ }^{1,5}$ and shortened rapid eye movement (REM) latency. ${ }^{1-3}$ However, investigations of the sleep disturbances associated with schizophrenia have yielded inconsistent results, possibly because of the heterogeneity of the disorder and differences in demographic characteristics and the clinical state, phase, and treatment of the illness.

Studies of spontaneous neural activity provide important information about brain function in patients with schizophrenia, and quantitative electroencephalography (EEG) is a useful technique for assessing brain function. Previous resting-awake EEGs performed in patients with schizophrenia have revealed increased theta and delta activity, decreased alpha activity, and

Received: June 14, 2016 Revised: August 7, 2016

Accepted: August 12, 2016 Available online: October 13, 2016

$\triangle$ Correspondence: Yu Jin Lee, MD, PhD

Department of Psychiatry, Seoul National University College of Medicine, 101 Daehak-ro, Jongno-gu, Seoul 03080, Republic of Korea

Tel: +82-2-2072-2456, Fax: +82-2-744-7241, E-mail: leeyj1203@gmail.com

(a) This is an Open Access article distributed under the terms of the Creative Commons Attribution Non-Commercial License (http://creativecommons.org/licenses/by$\mathrm{nc} / 4.0$ ) which permits unrestricted non-commercial use, distribution, and reproduction in any medium, provided the original work is properly cited. controversial results for beta activity. ${ }^{6}$ Sleep EEG is advantageous over waking EEG in that confounding factors related to attention, physical activity, and the presence of active symptoms are minimized. A few recent studies have used quantitative sleep EEG to assess patients with schizophrenia. Of those, some have reported reduced delta ${ }^{1,5,7}$ and sleep spindle activity ${ }^{8}$ and others have shown elevated beta activity. ${ }^{9}$ Delta waves are mediated by thalamo-cortical neurons, ${ }^{10}$ and sleep spindles are initiated by the thalamic reticular nucleus ${ }^{10,11}$ in the brain. Patients with schizophrenia have thalamo-cortical dysfunction, ${ }^{12-15}$ which may be related to the generation of delta waves and sleep spindles. Lower frequency $(<1 \mathrm{~Hz})$ delta waves during sleep may reflect relevant changes in the functional state of the thalamo-cortical network, ${ }^{16}$ so a prominent abnormality in the $0.5-1 \mathrm{~Hz}$ component is predicted in patients with schizophrenia. Increased beta activity has been suggested to reflect cortical excitation ${ }^{17}$ or tension and anxiety ${ }^{18}$ in patients with schizophrenia. Moreover, the negative symptoms of schizophrenia have been associated with decreased delta sleep, ${ }^{7,19}$ and positive symptoms have been associated with increased beta activity ${ }^{9}$

A limitation of most previous sleep micro structural studies of schizophrenia is that the EEG was recorded in only one $e^{5,9}$ or two regions. ${ }^{8,20}$ Moreover, few previous studies have used nocturnal polysomnography to exclude sleep disorders. EEG can 
detect cortical regional differences ${ }^{21}$ that may reflect brain function; thus, EEG patterns in patients with schizophrenia may differ depending on the cortical region considered. Thus, spectral analysis of the sleep EEG in several cortical regions may provide more information about functional changes in the brain of patients with schizophrenia than recording at one or two sites. Therefore, we used quantitative sleep EEG to compare activity in the bilateral frontal, central, and occipital areas in patients with schizophrenia and in healthy controls.

\section{METHODS}

\section{Study subjects}

We reviewed the medical records of 10 patients with schizophrenia who had undergone nocturnal polysomnography (Profusion PSG3, Compumedics, Abbottsford, VIC, Australia) at the Sleep and Chronobiology Center at Seoul National University Hospital. Based on interviews with a board-certified psychiatrist, we enrolled seven patients diagnosed with schizophrenia and three diagnosed with other psychotic disorders not due to a substance or known physiological condition according to the criteria of the International Classification of Diseases 10th revision. ${ }^{22}$ One patient was excluded because of severe obstructive sleep apnea; thus, a total of nine patients were included in the final analysis. Of those, one was medication naive, and eight were taking second-generation $(n=7)$ or first-generation $(n=1)$ antipsychotic drugs. The antipsychotic doses were converted to chlorpromazine equivalents. ${ }^{23}$ Three patients were taking concomitant sedatives (zolpidem $5 \mathrm{mg} /$ day, $\mathrm{n}=2$ and lorazepam $0.5 \mathrm{mg} /$ day, $\mathrm{n}=1$ ). The patients' medical records were used to retrospectively assess the severity of illness using the Clinical Global Impression scale for schizophrenia. We enrolled 10 age- and sex-matched healthy control subjects, based on a structured psychiatric interview, via advertisement. The control subjects underwent nocturnal polysomnography using the same protocol as that used for the patients with schizophrenia. Our study was approved by the Institutional Review Board of Seoul National University Hospital.

\section{Sleep recordings}

A standard EEG montage and referential derivations were used for nocturnal polysomnography. EEG was recorded at F3, F4, C3, C4, O1, and O2, using A1 and A2 as reference sites. The sleep variables monitored were time in bed, total sleep time, sleep efficiency, sleep latency, SWS latency, REM sleep latency, wake after sleep onset, the apnea-hypopnea index, and the percentage of each sleep stage relative to total sleep time. The polysomnographic data were scored by an experienced technician and physician, according to the American Acade- my of Sleep Medicinemanual. ${ }^{24}$

Quantitative spectral analysis was performed on each 5-s epoch after removing non-neurological EEG artifacts using the qEEG-PSA program (CIRUS, Camperdown, NSW, Australia). The absolute spectral power for non-REM (NREM) stages N2-3 was obtained in low frequency delta $(0.5-1 \mathrm{~Hz})$, delta $(0.5-4.5 \mathrm{~Hz})$, theta $(4.5-8 \mathrm{~Hz})$, alpha $(8-12 \mathrm{~Hz})$, sigma $(12-15 \mathrm{~Hz})$, beta $(15-32 \mathrm{~Hz})$, and gamma $(32-45 \mathrm{~Hz})$ frequency bands. The relative power for each EEG frequency band was expressed as a ratio of band energy to total power and used in the final analyses.

\section{Statistical analysis}

As the data did not consistently meet the criteria for a normal distribution, nonparametric statistical tests were conducted. The Mann-Whitney test was used to compare demographic characteristics, polysomnographic variables, and the spectral power of the EEG bands between groups. The effect of antipsychotic equivalent dosage on EEG spectral power was analyzed in the patient group with Spearman's correlation coefficient analysis. A p-value $<0.05$ was considered to indicate statistical significance. All statistical tests were conducted using the Statistical Package for Social Sciences version 18.0 for Windows (SPSS Inc., Chicago, IL, USA).

\section{RESULTS}

The participant demographic and clinical variables are shown in Table 1 . The polysomnographic variables of patients with schizophrenia and controls are shown in Table 2. The time in bed, sleep latency, and apnea-hypoxia index values were significantly higher in the patient than in the control group ( $\mathrm{p}=0.001,0.028$, and 0.035 , respectively). No significant differences were observed in total sleep time, total wake time, wake after sleep onset, and sleep efficiency, SWS latency, REM sleep latency, REM percentage, and N1, N2, and N3 percentages of total sleep time.

The quantitative EEG spectral activity during N2 and N3 stage sleep in patients with schizophrenia and controls is shown in Table 3 and 4, respectively. During N2 sleep, the 0.5$1 \mathrm{~Hz}$ activity was significantly lower and theta activity was significantly higher at F3, and beta activity was significantly higher at $\mathrm{O} 1$ in patients compared with controls. During N3, the alpha activity at $\mathrm{O} 1$ was significantly higher in the patients than in controls. No other significant differences were observed between groups.

The correlations between antipsychotic equivalent dosage and spectral power of the EEG with significant between-group differences are shown in Table 5. Only beta activity at O1 during N2 sleep was inversely correlated with antipsychotic equiv- 
alent doses $(\mathrm{Rho}=-0.767, \mathrm{p}=0.016)$.

\section{DISCUSSION}

We compared EEG sleep patterns in patients with schizophrenia and healthy controls. We found that the patients spent more time in bed than controls, but had difficulty getting to sleep. During N2, we observed significantly lower $0.5-1 \mathrm{~Hz}$ activity and higher theta activity at F3, higher beta activity at $\mathrm{O} 1$ in the patients compared with control subjects. During N3, alpha activity at $\mathrm{O} 1$ was significantly higher in patients with schizophrenia than in the control group.

Previous studies have found abnormal EEG sleep patterns in patients with schizophrenia. Keshavan et al. ${ }^{5}$ reported lower delta activity in the right central area of patients with schizophrenia during NREM sleep, and Göder et al. ${ }^{25}$ observed lower delta activity in the bilateral occipital area of patients during SWS. Our findings that $0.5-1 \mathrm{~Hz}$ activity was significantly lower in patients than in controls in the left frontal regions during N2 were partially consistent with those of previous studies. Tekell et al. ${ }^{9}$ reported increased beta activity in the left central region of patients with schizophrenia during NREM sleep, whereas we observed increased beta activity in the left occipital region of patients with schizophrenia only during N2. Our failure to find significant between-group differences in more cortical areas and sleep stages may be explained by our small sample size: we found consistent, but non-significant, trends toward lower delta and higher beta power in patients compared with controls in other cortical regions and sleep stages. Further studies with larger samples are needed to confirm and extend our results. In contrast to previous findings showing no change ${ }^{9}$ or a decrease in theta activity, ${ }^{5}$ we found increased theta activity in the left frontal region of patients with schizophrenia. Moreover, we observed increased alpha activity in the patients. To our knowledge, our study is the first to report EEG changes in alpha activity during sleep in patients with schizophrenia.

The prefrontal cortex is thought to be important for generating synchronized SWSactivity. ${ }^{26}$ Several studies have demonstrated frontal lobe dysfunction in patients with schizophre-

Table 1. Demographic and clinical variables in patients with schizophrenia and control subjects

\begin{tabular}{|c|c|c|c|c|c|c|c|}
\hline \multirow{2}{*}{ Characteristics } & \multicolumn{3}{|c|}{ Patients with schizophrenia $(\mathrm{N}=9)$} & \multicolumn{3}{|c|}{ Controls $(\mathrm{N}=10)$} & \multirow{2}{*}{$\mathrm{p}^{*}$} \\
\hline & Mean & SD & Range & Mean & $\mathrm{SD}$ & Range & \\
\hline Age (years) & 30.0 & 13.6 & $18-63$ & 30.3 & 8.4 & $22-46$ & 0.549 \\
\hline Male/female & \multicolumn{3}{|c|}{ 6/3 (male: 66.7\%) } & \multicolumn{3}{|c|}{$7 / 3$ (male: $70 \%$ ) } & \\
\hline BMI $\left(\mathrm{kg} / \mathrm{m}^{2}\right)$ & 25.2 & 4.5 & $19.7-32.9$ & 22.5 & 2.2 & $19.1-26.1$ & 0.182 \\
\hline Duration of illness (years) & 9.0 & 13.4 & $1-44$ & & & & \\
\hline CGI & 2.9 & 0.8 & $2-4$ & & & & \\
\hline Antipsychotic medication $\operatorname{dose}^{\dagger}$ (mg/day) & 269.9 & 324.0 & $(0-1066.7)$ & & & & \\
\hline
\end{tabular}

*the Mann-Whitney test between patients with schizophrenia and controls, ${ }^{\dagger}$ chlorpromazine-equivalent dose calculated using published conversions for antipsychotics. ${ }^{22}$ BMI: body mass index, CGI: clinical global severity index

Table 2. Polysomnographic variables in patients with schizophrenia and control subjects

\begin{tabular}{|c|c|c|c|c|c|c|c|}
\hline \multirow{2}{*}{ Variables } & \multicolumn{3}{|c|}{ Patients with schizophrenia $(\mathrm{N}=9)$} & \multicolumn{3}{|c|}{ Controls $(\mathrm{N}=10)$} & \multirow{2}{*}{$\mathrm{p}^{\dagger}$} \\
\hline & Mean & SD & Range & Mean & SD & Range & \\
\hline Time in bed (min) & 524 & 25.7 & $487.5-560.5$ & 481.6 & 19.2 & $449.0-505.0$ & $0.001^{*}$ \\
\hline Total sleep time (min) & 404.1 & 80.9 & $252.5-472.5$ & 422.8 & 51.4 & $300.0-483.0$ & 0.905 \\
\hline Sleep efficiency (\%) & 77.0 & 14.7 & $51.8-96.4$ & 87.9 & 10.5 & $59.4-94.8$ & 0.065 \\
\hline Sleep onset latency (min) & 54.4 & 38.8 & $7.5-105.0$ & 15.2 & 7.2 & $7.5-26.5$ & $0.028^{*}$ \\
\hline Slow-wave sleep latency (min) & 24.4 & 19.3 & $3.5-58.0$ & 15.3 & 3.8 & $11.5-24.0$ & 0.156 \\
\hline REM latency (min) & 115.9 & 85.4 & $0.0-239.0$ & 128.2 & 59.2 & $82.0-277.5$ & 0.842 \\
\hline Wake after sleep onset (min) & 65.4 & 70.6 & $5.5-186.0$ & 43.6 & 49.3 & $14.5-178.5$ & 1.000 \\
\hline N1 (\%) & 13.3 & 10.4 & $4.9-32.6$ & 11.2 & 7.9 & $2.7-29.3$ & 0.780 \\
\hline N2 (\%) & 51.0 & 12.7 & $38.6-59.1$ & 54.1 & 7.9 & $39.3-63.5$ & 0.278 \\
\hline N3 (\%) & 20.0 & 10.8 & $4.0-35.4$ & 12.6 & 6.9 & $3.7-28.4$ & 0.113 \\
\hline REM (\%) & 15.7 & 10.8 & $0.0-30.6$ & 22.1 & 2.9 & $18.5-25.5$ & 0.278 \\
\hline Apnea-hypopnea index & 5.0 & 3.7 & $0.4-12.0$ & 1.6 & 1.8 & $0.1-5.5$ & $0.035^{*}$ \\
\hline
\end{tabular}

${ }^{*} \mathrm{p}<0.05$, the Mann-Whitney test between patients with schizophrenia and controls. REM: rapid eye movement 
Table 3. Comparison of EEG spectral activity during N2 sleep in patients with schizophrenia and control subjects

\begin{tabular}{|c|c|c|c|c|c|c|c|}
\hline \multirow{2}{*}{ Variables } & \multicolumn{3}{|c|}{ Patients with schizophrenia $(\mathrm{N}=9)$} & \multicolumn{3}{|c|}{ Controls $(\mathrm{N}=10)$} & \multirow{2}{*}{$\mathrm{p}^{\dagger}$} \\
\hline & Mean & $\mathrm{SD}$ & Range & Mean & $\mathrm{SD}$ & Range & \\
\hline \multicolumn{8}{|l|}{ F3 } \\
\hline Low delta $(0.5-1 \mathrm{~Hz})$ & 39.8 & 10.9 & $20.0-51.3$ & 52.1 & 7.9 & $39.7-70.0$ & 0.010 \\
\hline Delta $(0.5-4.5 \mathrm{~Hz})$ & 84.9 & 6.6 & $72.4-92.2$ & 90.1 & 2.4 & $87.5-95.4$ & 0.053 \\
\hline Theta $(4.5-8 \mathrm{~Hz})$ & 6.8 & 2.6 & $4.6-12.9$ & 4.9 & 1.6 & $2.3-8.4$ & $0.035^{*}$ \\
\hline Alpha $(8-12 \mathrm{~Hz})$ & 4.1 & 2.6 & $1.7-9.6$ & 2.5 & 0.8 & $1.0-3.7$ & 0.095 \\
\hline Sigma $(12-15 \mathrm{~Hz})$ & 1.7 & 1.4 & $0.5-2.4$ & 1.1 & 0.4 & $0.4-1.7$ & 0.400 \\
\hline $\operatorname{Beta}(15-32 \mathrm{~Hz})$ & 2.1 & 1.4 & $0.7-4.7$ & 1.2 & 0.5 & $0.7-2.7$ & 0.133 \\
\hline Gamma $(32-45 \mathrm{~Hz})$ & 0.4 & 0.4 & $0.1-1.5$ & 0.2 & 0.1 & $0.2-0.4$ & 0.356 \\
\hline \multicolumn{8}{|l|}{$\mathrm{F} 4$} \\
\hline Low delta $(0.5-1 \mathrm{~Hz})$ & 41.8 & 51.4 & $20.8-63.1$ & 14.7 & 14.1 & $23.0-68.8$ & 0.156 \\
\hline Delta $(0.5-4.5 \mathrm{~Hz})$ & 84.9 & 7.2 & $73.9-92.8$ & 89.2 & 5.1 & $78.2-95.1$ & 0.315 \\
\hline Theta $(4.5-8 \mathrm{~Hz})$ & 7.2 & 3.4 & $3.5-12.2$ & 5.6 & 4.0 & $2.4-16.2$ & 0.211 \\
\hline Alpha $(8-12 \mathrm{~Hz})$ & 4.1 & 2.7 & $1.5-9.4$ & 2.4 & 1.0 & $1.1-4.1$ & 0.211 \\
\hline Sigma $(12-15 \mathrm{~Hz})$ & 1.5 & 1.2 & $04-4.3$ & 1.2 & 0.6 & $0.5-2.1$ & 0.905 \\
\hline Beta $(15-32 \mathrm{~Hz})$ & 1.9 & 1.1 & $0.6-3.7$ & 1.3 & 0.6 & $0.7-2.7$ & 0.400 \\
\hline Gamma $(32-45 \mathrm{~Hz})$ & 0.3 & 0.2 & $0.1-0.7$ & 0.2 & 0.1 & $0.1-0.5$ & 0.447 \\
\hline \multicolumn{8}{|l|}{ C3 } \\
\hline Low delta $(0.5-1 \mathrm{~Hz})$ & 42.9 & 10.1 & $30.1-59.7$ & 51.1 & 8.7 & $38.1-68.4$ & 0.095 \\
\hline Delta $(0.5-4.5 \mathrm{~Hz})$ & 83.7 & 6.9 & $70.3-91.3$ & 88.5 & 3.2 & $82.0-93.3$ & 0.053 \\
\hline Theta $(4.5-8 \mathrm{~Hz})$ & 7.2 & 3.0 & $4.7-14.1$ & 5.8 & 2.6 & $3.2-12.3$ & 0.133 \\
\hline Alpha $(8-12 \mathrm{~Hz})$ & 4.2 & 2.7 & $1.8-9.6$ & 2.6 & 0.7 & $1.5-3.7$ & 0.133 \\
\hline Sigma $(12-15 \mathrm{~Hz})$ & 2.0 & 1.7 & $0.5-6.3$ & 1.4 & 0.5 & $0.6-2.2$ & 0.447 \\
\hline Beta $(15-32 \mathrm{~Hz})$ & 2.4 & 1.2 & $0.8-4.0$ & 1.5 & 0.5 & $0.9-2.7$ & 0.095 \\
\hline Gamma $(32-45 \mathrm{~Hz})$ & 0.4 & 0.3 & $0.1-0.8$ & 0.2 & 0.1 & $0.2-0.4$ & 0.211 \\
\hline \multicolumn{8}{|l|}{$\mathrm{C} 4$} \\
\hline Low delta $(0.5-1 \mathrm{~Hz})$ & 42.5 & 15.1 & $22.6-65.9$ & 49.8 & 11.1 & $28.2-65.2$ & 0.400 \\
\hline Delta $(0.5-4.5 \mathrm{~Hz})$ & 83.9 & 7.3 & $70.7-92.2$ & 87.6 & 4.4 & $79.8-93.7$ & 0.278 \\
\hline Theta $(4.5-8 \mathrm{~Hz})$ & 7.7 & 3.5 & $3.7-12.9$ & 6.2 & 3.4 & $3.0-14.6$ & 0.356 \\
\hline Alpha $(8-12 \mathrm{~Hz})$ & 4.3 & 2.8 & $1.6-10.1$ & 2.7 & 1.1 & $1.4-4.5$ & 0.278 \\
\hline Sigma $(12-15 \mathrm{~Hz})$ & 1.8 & 1.5 & $0.5-5.6$ & 1.5 & 0.5 & $0.7-2.1$ & 0.842 \\
\hline Beta $(15-32 \mathrm{~Hz})$ & 2.1 & 1.1 & $0.6-3.8$ & 1.8 & 0.9 & $0.9-3.5$ & 0.604 \\
\hline Gamma $(32-45 \mathrm{~Hz})$ & 0.3 & 0.2 & $0.1-0.7$ & 0.3 & 0.2 & $0.2-0.7$ & 0.780 \\
\hline \multicolumn{8}{|l|}{$\mathrm{O} 1$} \\
\hline Low delta $(0.5-1 \mathrm{~Hz})$ & 49.8 & 15.6 & $21.7-69.0$ & 61.8 & 10.0 & $40.0-74.2$ & 0.095 \\
\hline Delta $(0.5-4.5 \mathrm{~Hz})$ & 84.1 & 8.7 & $65.7-91.8$ & 90.9 & 3.6 & $84.7-96.3$ & 0.053 \\
\hline Theta $(4.5-8 \mathrm{~Hz})$ & 7.5 & 3.5 & $3.4-12.4$ & 4.5 & 1.6 & $1.8-6.8$ & 0.079 \\
\hline Alpha $(8-12 \mathrm{~Hz})$ & 3.8 & 2.4 & $1.7-9.6$ & 2.1 & 1.0 & $0.8-3.7$ & 0.053 \\
\hline Sigma (12-15 Hz) & 1.8 & 1.9 & $0.6-6.7$ & 1.1 & 0.7 & $0.3-2.8$ & 0.278 \\
\hline Beta $(15-32 \mathrm{~Hz})$ & 2.4 & 1.7 & $1.0-5.6$ & 1.3 & 0.6 & $0.6-2.6$ & $0.035^{*}$ \\
\hline Gamma $(32-45 \mathrm{~Hz})$ & 0.4 & 0.3 & $0.2-0.9$ & 0.2 & 0.1 & $0.2-0.5$ & 0.095 \\
\hline \multicolumn{8}{|l|}{$\mathrm{O} 2$} \\
\hline Low delta $(0.5-1 \mathrm{~Hz})$ & 46.4 & 20.4 & $10.4-70.2$ & 46.4 & 56.6 & $31.1-72.1$ & 0.243 \\
\hline Delta $(0.5-4.5 \mathrm{~Hz})$ & 82.5 & 10.8 & $64.5-93.9$ & 88.5 & 4.9 & $80.5-94.2$ & 0.356 \\
\hline Theta $(4.5-8 \mathrm{~Hz})$ & 8.8 & 5.8 & $3.4-18.2$ & 5.7 & 3.4 & $2.2-13.8$ & 0.400 \\
\hline Alpha $(8-12 \mathrm{~Hz})$ & 4.3 & 3.1 & $1.4-9.2$ & 2.4 & 1.2 & $1.0-4.0$ & 0.182 \\
\hline Sigma $(12-15 \mathrm{~Hz})$ & 1.8 & 1.6 & $0.4-5.8$ & 1.3 & 0.8 & $0.4-2.8$ & 0.905 \\
\hline Beta $(15-32 \mathrm{~Hz})$ & 2.2 & 1.4 & $0.7-5.2$ & 1.7 & 0.6 & $0.9-2.7$ & 0.661 \\
\hline Gamma $(32-45 \mathrm{~Hz})$ & 0.4 & 0.2 & $0.2-0.9$ & 0.3 & 0.1 & $0.2-0.6$ & 0.549 \\
\hline
\end{tabular}

${ }^{*} \mathrm{p}<0.05,{ }^{\text {the }}$ Mann-Whitney test between patients with schizophrenia and controls. EEG: electroencephalography 
SM Oh et al.

Table 4. Comparisons of EEG spectral activity during N3 sleep between patients with schizophrenia and control subjects

\begin{tabular}{|c|c|c|c|c|c|c|c|}
\hline \multirow{2}{*}{ Variables } & \multicolumn{3}{|c|}{ Patients with schizophrenia $(\mathrm{N}=9)$} & \multicolumn{3}{|c|}{ Controls $(\mathrm{N}=10)$} & \multirow{2}{*}{$\mathrm{p}^{\dagger}$} \\
\hline & Mean & $\mathrm{SD}$ & Range & Mean & $\mathrm{SD}$ & Range & \\
\hline \multicolumn{8}{|l|}{ F3 } \\
\hline Low delta $(0.5-1 \mathrm{~Hz})$ & 46.5 & 9.6 & $30.5-61.9$ & 50.1 & 16.2 & $10.6-67.6$ & 0.315 \\
\hline Delta $(0.5-4.5 \mathrm{~Hz})$ & 91.2 & 4.2 & $83.5-94.7$ & 92.0 & 6.6 & $73.8-96.1$ & 0.211 \\
\hline Theta $(4.5-8 \mathrm{~Hz})$ & 4.4 & 2.1 & $2.8-9.5$ & 4.9 & 5.5 & $2.2-20.2$ & 0.315 \\
\hline Alpha $(8-12 \mathrm{~Hz})$ & 2.4 & 1.5 & $1.2-5.0$ & 1.5 & 0.6 & $0.8-3.1$ & 0.133 \\
\hline Sigma $(12-15 \mathrm{~Hz})$ & 0.8 & 0.6 & $0.5-5.0$ & 0.7 & 0.6 & $0.3-2.4$ & 0.661 \\
\hline Beta $(15-32 \mathrm{~Hz})$ & 1.0 & 0.6 & $0.5-2.1$ & 0.8 & 0.5 & $0.5-2.2$ & 0.604 \\
\hline Gamma $(32-45 \mathrm{~Hz})$ & 0.2 & 0.1 & $0.1-0.5$ & 0.1 & 0.0 & $0.1-0.2$ & 0.661 \\
\hline \multicolumn{8}{|l|}{$\mathrm{F} 4$} \\
\hline Low delta $(0.5-1 \mathrm{~Hz})$ & 48.6 & 11.7 & $29.1-65.3$ & 52.8 & 17.6 & $10.3-70.9$ & 0.315 \\
\hline Delta $(0.5-4.5 \mathrm{~Hz})$ & 91.5 & 3.9 & $84.0-95.7$ & 92.5 & 6.6 & $74.3-96.4$ & 0.243 \\
\hline Theta $(4.5-8 \mathrm{~Hz})$ & 4.4 & 2.2 & $2.3-9.3$ & 4.6 & 5.4 & $1.8-19.7$ & 0.156 \\
\hline Alpha $(8-12 \mathrm{~Hz})$ & 2.2 & 1.4 & $1.1-4.8$ & 1.3 & 0.4 & $0.8-1.9$ & 0.156 \\
\hline Sigma $(12-15 \mathrm{~Hz})$ & 0.7 & 0.5 & $0.3-1.8$ & 0.7 & 0.6 & $0.3-2.4$ & 0.780 \\
\hline Beta $(15-32 \mathrm{~Hz})$ & 0.9 & 0.6 & $0.4-2.3$ & 0.8 & 0.5 & $0.5-2.2$ & 0.497 \\
\hline Gamma $(32-45 \mathrm{~Hz})$ & 0.2 & 0.1 & $0.1-0.3$ & 0.1 & 0.0 & $0.1-0.2$ & 0.780 \\
\hline \multicolumn{8}{|l|}{$\mathrm{C} 3$} \\
\hline Low delta $(0.5-1 \mathrm{~Hz})$ & 49.8 & 11.7 & $32.0-69.5$ & 49.7 & 16.8 & $10.2-73.4$ & 0.905 \\
\hline Delta $(0.5-4.5 \mathrm{~Hz})$ & 90.7 & 4.9 & $81.8-94.6$ & 91.3 & 7.0 & $72.3-95.8$ & 0.356 \\
\hline Theta $(4.5-8 \mathrm{~Hz})$ & 4.6 & 2.4 & $2.6-10.3$ & 5.3 & 5.7 & $2.1-21.3$ & 0.549 \\
\hline Alpha $(8-12 \mathrm{~Hz})$ & 2.3 & 1.6 & $1.2-5.2$ & 1.5 & 0.7 & $0.9-2.9$ & 0.211 \\
\hline Sigma $(12-15 \mathrm{~Hz})$ & 1.0 & 0.8 & $0.4-2.7$ & 0.8 & 0.6 & $0.4-2.4$ & 0.356 \\
\hline Beta $(15-32 \mathrm{~Hz})$ & 1.2 & 0.7 & $0.5-2.5$ & 1.0 & 0.6 & $0.5-2.4$ & 0.447 \\
\hline Gamma $(32-45 \mathrm{~Hz})$ & 0.2 & 0.1 & $0.1-0.5$ & 0.2 & 0.0 & $0.1-0.3$ & 0.720 \\
\hline \multicolumn{8}{|l|}{$\mathrm{C} 4$} \\
\hline Low delta $(0.5-1 \mathrm{~Hz})$ & 50.5 & 14.7 & $30.4-73.5$ & 54.4 & 14.8 & $21.7-73.8$ & 0.497 \\
\hline Delta $(0.5-4.5 \mathrm{~Hz})$ & 90.5 & 5.3 & $82,3-95.4$ & 92.9 & 4.8 & $80.1-98.0$ & 0.604 \\
\hline Theta $(4.5-8 \mathrm{~Hz})$ & 4.9 & 2.6 & $2.2-10.0$ & 4.2 & 3.8 & $1.0-14.7$ & 0.400 \\
\hline Alpha $(8-12 \mathrm{~Hz})$ & 2.3 & 1.6 & $1.0-4.6$ & 1.3 & 0.4 & $0.4-2.0$ & 0.400 \\
\hline Sigma $(12-15 \mathrm{~Hz})$ & 1.0 & 0.9 & $0.4-3.1$ & 0.6 & 0.4 & $0.2-1.7$ & 0.842 \\
\hline Beta $(15-32 \mathrm{~Hz})$ & 1.2 & 0.9 & $0.5-3.6$ & 0.9 & 0.4 & $0.3-1.8$ & 0.549 \\
\hline Gamma $(32-45 \mathrm{~Hz})$ & 0.2 & 0.1 & $0.1-0.5$ & 0.2 & 0.1 & $0.1-0.3$ & 0.842 \\
\hline \multicolumn{8}{|l|}{$\mathrm{O} 1$} \\
\hline Low delta $(0.5-1 \mathrm{~Hz})$ & 52.5 & 12.3 & $30.7-72.2$ & 53.6 & 14.8 & $11.8-77.1$ & 0.095 \\
\hline Delta $(0.5-4.5 \mathrm{~Hz})$ & 88.0 & 8.5 & $68.8-95.1$ & 91.7 & 6.3 & $74.9-97.7$ & 0.211 \\
\hline Theta $(4.5-8 \mathrm{~Hz})$ & 5.4 & 2.2 & $2.2-8.9$ & 5.1 & 5.1 & $1.2-19.1$ & 0.211 \\
\hline Alpha $(8-12 \mathrm{~Hz})$ & 2.7 & 2.3 & $0.9-8.0$ & 1.3 & 0.5 & $0.5-2.3$ & $0.043^{*}$ \\
\hline Sigma (12-15 Hz) & 1.5 & 1.8 & $0.4-5.7$ & 0.7 & 0.6 & $0.2-2.4$ & 0.400 \\
\hline Beta $(15-32 \mathrm{~Hz})$ & 2.0 & 2.3 & $0.7-7.5$ & 0.9 & 0.5 & $0.3-2.2$ & 0.182 \\
\hline Gamma $(32-45 \mathrm{~Hz})$ & 0.4 & 0.4 & $0.1-1.2$ & 0.2 & 0.1 & $0.1-0.3$ & 0.182 \\
\hline \multicolumn{8}{|l|}{$\mathrm{O} 2$} \\
\hline Low delta $(0.5-1 \mathrm{~Hz})$ & 53.6 & 14.8 & $34.5-73.7$ & 58.9 & 19.5 & $10.6-74.1$ & 0.400 \\
\hline Delta $(0.5-4.5 \mathrm{~Hz})$ & 88.7 & 7.4 & $73.2-95.4$ & 92.1 & 7.2 & $72.1-97.9$ & 0.243 \\
\hline Theta $(4.5-8 \mathrm{~Hz})$ & 5.7 & 3.0 & $2.2-9.2$ & 4.8 & 5.9 & $1.1-21.3$ & 0.211 \\
\hline Alpha $(8-12 \mathrm{~Hz})$ & 2.6 & 1.9 & $0.9-6.8$ & 1.2 & 0.4 & $0.4-1.6$ & 0.079 \\
\hline Sigma $(12-15 \mathrm{~Hz})$ & 1.2 & 1.3 & $0.4-4.3$ & 0.7 & 0.6 & $0.2-2.4$ & 0.447 \\
\hline Beta $(15-32 \mathrm{~Hz})$ & 1.6 & 1.9 & $0.7-6.7$ & 1.0 & 0.6 & $0.3-2.4$ & 0.661 \\
\hline Gamma $(32-45 \mathrm{~Hz})$ & 0.3 & 0.2 & $0.2-0.9$ & 0.2 & 0.1 & $0.1-0.3$ & 0.400 \\
\hline
\end{tabular}

${ }^{*} \mathrm{p}<0.05,{ }^{\dagger}$ the Mann-Whitney test between patients with schizophrenia and controls. EEG: electroencephalography 
Table 5. Correlations between antipsychotic equivalent dosage ${ }^{\ddagger}$ and quantitative EEG spectral activity in patients with schizophrenia

\begin{tabular}{ccccc}
\hline Sleep stage & & Variables & Rho & $\mathrm{p}^{\dagger}$ \\
\hline N2 & F3 & $0.5-1 \mathrm{~Hz}$ & 0.383 & 0.308 \\
& F3 & Theta & -0.217 & 0.576 \\
& O1 & Beta & -0.767 & $0.016^{*}$ \\
N3 & O1 & Alpha & -0.383 & 0.308 \\
\hline
\end{tabular}

${ }^{*} \mathrm{p}<0.05$, ${ }^{\dagger}$ Spearman’s coefficient of correlation, ${ }^{\ddagger}$ chlorpromazineequivalent dose calculated using published conversions for antipsychotics. ${ }^{22}$ EEG: electroencephalography

nia, ${ }^{27,28}$ which may be associated with decreased EEG slow wave activity during sleep, particularly in the frontal region. In addition, thalamo-cortical disturbances in patients with schizophrenia $^{12-15}$ may be associated with decreased delta wave activity.

Antipsychotic medications have distinct effects on sleep ${ }^{29}$; thus, medication may have affected the EEG sleep patterns of our patients. In our study, no significant correlation was found between antipsychotic equivalent dosage and low frequency delta $(0.5-1 \mathrm{~Hz})$, theta, or alpha activity. Beta activity during $\mathrm{N} 2$ sleep was inversely correlated with antipsychotic equivalent dosage. Thus, the increase in beta activity in patients with schizophrenia may not be explained by an effect of antipsychotic medication.

The most striking finding of our study was that all of the significant differences between the patient and control groups were observed in the left cortical hemisphere (F3, O1). Although we had no data concerning the right or left-handedness of our subjects, it may be that micro structural changes in the sleep patterns of patients with schizophrenia are affected by brain hemisphere dominance. This notion is supported by a previous imaging study showing decreased leftward functional hemispheric lateralization and leftward asymmetry in the thalamus in patients with schizophrenia. ${ }^{30}$ However, asleep study using period amplitude analyses found a more marked decrease in delta wave count in the right than in the left frontal and central regions in right-handed patients with schizophrenia. ${ }^{7}$ Given these discrepancies, further studies are needed to clarify the significance of brain hemisphere dominance on sleep.

Although we found no significant differences in sleep macrostructure between patients with schizophrenia and healthy controls, the sleep microstructures were significantly different between groups. Decreased slow wave EEG activity and increased alpha and beta activity during sleep are indicators of cerebral dysfunction in patients with schizophrenia, who tend to sleep less deeply and wake after sleep onset more often than healthy subjects. Moreover, such sleep disruptions may be associated with a decline in cognitive function and poor emotional regulation in patients with schizophrenia. ${ }^{31}$ Particularly, de- creased activity in the low delta $0.5-1 \mathrm{~Hz}$ frequency which involved in memory consolidation during sleep in young adults $^{32}$ may be related with cognitive impairment in patients with schizophrenia.

Our study has several limitations. First, our sample size was small. Second, schizophrenia is a heterogeneous condition with differences in clinical state, phase, and treatment. Furthermore, we could not rule out pharmacological effects of various antipsychotic ${ }^{33}$ and other medications ${ }^{34,35}$ on the EEG findings. Third, the higher apnea-hypopnea index (AHI) of patients compared with that in control subjects may have affected their sleep structure. Because of the restriction of nonparametric tests, we could not adjust for the confounding effect of the AHI.

In conclusion, using EEG spectral analysis, we identified distinctive differences in the sleep microstructure of patients with schizophrenia compared with healthy controls. Decreased low frequency delta $(0.5-1 \mathrm{~Hz})$ activity and increased beta and alpha activity during sleep in the patients were indicators of sleep disturbances that may be related to brain dysfunction or the effect of medication. Furthermore, we found different topographical EEG patterns according to cortical region. Further studies with larger study populations are needed to confirm the specificity of our preliminary findings and adjust for the confounding effect of sleep apnea. Pathophysiological significance of the regional differences in the EEG pattern needs to be studied as well.

\section{REFERENCES}

1. Hiatt JF, Floyd TC, Katz PH, Feinberg I. Further evidence of abnormal non-rapid-eye-movement sleep in schizophrenia. Arch Gen Psychiatry 1985;42:797-802.

2. Tandon R, Shipley JE, Taylor S, Greden JF, Eiser A, DeQuardo J, et al. Electroencephalographic sleep abnormalities in schizophrenia. Relationship to positive/negative symptoms and prior neuroleptic treatment. Arch Gen Psychiatry 1992;49:185-194.

3. Hudson JI, Lipinski JF, Keck PE Jr, Aizley HG, Vuckovic A, Zierk KC, et al. Polysomnographic characteristics of schizophrenia in comparison with mania and depression. Biol Psychiatry 1993;34:191-193.

4. Lauer CJ, Schreiber W, Pollmächer T, Holsboer F, Krieg JC. Sleep in schizophrenia: a polysomnographic study on drug-naive patients. Neuropsychopharmacology 1997;16:51-60.

5. Keshavan MS, Reynolds CF 3rd, Miewald JM, Montrose DM, Sweeney JA, Vasko RC, et al. Delta sleep deficits in schizophrenia: evidence from automated analyses of sleep data. Arch Gen Psychiatry 1998;55: 443-448.

6. Boutros NN, Arfken C, Galderisi S, Warrick J, Pratt G, Iacono W. The status of spectral EEG abnormality as a diagnostic test for schizophrenia. Schizophr Res 2008;99:225-237.

7. Sekimoto M, Kato M, Watanabe T, Kajimura N, Takahashi K. Cortical regional differences of delta waves during all-night sleep in schizophrenia. Schizophr Res 2011;126:284-290.

8. Ferrarelli F, Huber R, Peterson MJ, Massimini M, Murphy M, Riedner BA, et al. Reduced sleep spindle activity in schizophrenia patients. Am J Psychiatry 2007;164:483-492.

9. Tekell JL, Hoffmann R, Hendrickse W, Greene RW, Rush AJ, Armitage 
R. High frequency EEG activity during sleep: characteristics in schizophrenia and depression. Clin EEG Neurosci 2005;36:25-35.

10. Steriade M, McCormick DA, Sejnowski TJ. Thalamocortical oscillations in the sleeping and aroused brain. Science 1993;262:679-685.

11. Steriade M. Grouping of brain rhythms in corticothalamic systems. Neuroscience 2006;137:1087-1106.

12. Andreasen NC, Arndt S, Swayze V 2nd, Cizadlo T, Flaum M, O'Leary $\mathrm{D}$, et al. Thalamic abnormalities in schizophrenia visualized through magnetic resonance image averaging. Science 1994;266:294-298.

13. Tononi G, Edelman GM. Schizophrenia and the mechanisms of conscious integration. Brain Res Brain Res Rev 2000;31:391-400.

14. Benes FM, Berretta S. GABAergic interneurons: implications for understanding schizophrenia and bipolar disorder. Neuropsychopharmacology 2001;25:1-27.

15. Volk DW, Lewis DA. Impaired prefrontal inhibition in schizophrenia: relevance for cognitive dysfunction. Physiol Behav 2002;77:501-505.

16. Massimini M, Rosanova M, Mariotti M. EEG slow $(\sim 1 \mathrm{~Hz})$ waves are associated with nonstationarity of thalamo-cortical sensory processing in the sleeping human. J Neurophysiol 2003;89:1205-1213.

17. Miyauchi T, Tanaka K, Hagimoto H, Miura T, Kishimoto H, Matsushita M. Computerized EEG in schizophrenic patients. Biol Psychiatry 1990;28:488-494.

18. Gattaz WF, Mayer S, Ziegler P, Platz M, Gasser T. Hypofrontality on topographic EEG in schizophrenia. Eur Arch Psychiatry Clin Neurosci 1992;241:328-332.

19. Keshavan MS, Miewald J, Haas G, Sweeney J, Ganguli R, Reynolds CF. Slow-wave sleep and symptomatology in schizophrenia and related psychotic disorders. J Psychiatr Res 1995;29:303-314.

20. Sekimoto M, Kato M, Watanabe T, Kajimura N, Takahashi K. Reduced frontal asymmetry of delta waves during all-night sleep in schizophrenia. Schizophr Bull 2007;33:1307-1311.

21. Sekimoto M, Kato M, Kajimura N, Watanabe T, Takahashi K, Okuma T. Asymmetric interhemispheric delta waves during all-night sleep in humans. Clin Neurophysiol 2000;111:924-928.

22. Organization WH. The ICD-10 Classification of Mental and Behavioural Disorders: Clinical Descriptions and Diagnostic Guidelines. Geneva: World Health Organization; 1992.

23. Leucht S, Samara M, Heres S, Patel MX, Woods SW, Davis JM. Dose equivalents for second-generation antipsychotics: the minimum effec- tive dose method. Schizophr Bull 2014;40:314-326.

24. Berry RB, Brooks R, Gamaldo CE, Harding SM, Marcus CL, Vaughn BV. The AASM manual for the scoring of sleep and associated events. Rules, Terminology and Technical Specifications, Darien: American Academy of Sleep Medicine, 2012.

25. Göder R, Aldenhoff M, Boigs M, Braun S, Koch J, Fritzer G. Delta power in sleep in relation to neuropsychological performance in healthy subjects and schizophrenia patients. J Neuropsychiatry Clinical Neurosci 2006;18:529-535.

26. Kryger MH, Roth T, Dement WC. Principles and Practice of Sleep Medicine (3rd ed). In: Jones B. Basic mechanisms of sleep-wake states. Philadelphia: PS WB Saunders, 2000, p.136-153.

27. Tan HY, Choo WC, Fones CS, Chee MW. fMRI study of maintenance and manipulation processes within working memory in first-episode schizophrenia. Am J Psychiatry 2005;162:1849-1958.

28. Molina V, Sanz J, Muñoz F, Casado P, Hinojosa JA, Sarramea F, et al. Dorsolateral prefrontal cortex contribution to abnormalities of the P300 component of the event-related potential in schizophrenia. Psychiatry Res 2005;140:17-26.

29. Giménez S, Clos S, Romero S, Grasa E, Morte A. Barbanoj MJ. Effects of olanzapine, risperidone and haloperidol on sleep after a single oral morning dose in healthy volunteers. Psychopharmacology (Berl) 2007; 190:507-516.

30. Okada N, Fukunaga M, Yamashita F, Koshiyama D, Yamamori H, Ohi $\mathrm{K}$, et al. Abnormal asymmetries in subcortical brain volume in schizophrenia. Mol Psychiatry 2016 [Epub ahead of print].

31. Walker MP. The role of sleep in cognition and emotion. Ann N Y Acad Sci 2009;1156:168-197.

32. Marshall L, Helgadottir H, Molle M, Born J. Boosting slow oscillations during sleep potentiates memory. Nature 2006;444:610-613.

33. Monti JM, Monti D. Sleep in schizophrenia patients and the effects of antipsychotic drugs. Sleep Med Rev 2004;8:133-148.

34. Bastien CH, LeBlanc M, Carrier J, Morin CM. Sleep EEG power spectra, insomnia, and chronic use of benzodiazepines. Sleep 2003;26:313317.

35. Monti JM, Alvarino F, Monti D. Conventional and power spectrum analysis of the effects of zolpidem on sleep EEG in patients with chronic primary insomnia. Sleep 2000;23:1075-1084. 